\section{Capitation of Radiology Practice in a Managed Care Environment in the USA and South Africa : Johannesburg seminar 2-3 March 1996}

\section{Paul F Gross}

Director Health Group Strategies Pty Ltd (Australia) $n$ the managed care environment entrenched or emerging, respectively, in the United States and South Africa, the use of capitation payments to larger networks of radiology practices seems likely to accelerate with increases in teleradiology, horizontal and vertical integration of health services, and the demand for utilisation management and outcomes measurement.

The common views of leaders in radiology in two nations as diverse as the United States and South Africa are instructive. Both can see that the major payers (the large HMO in the United States and either the new National Government or the large medical schemes organisations now offering indemnity insurance in South Africa) have a common problem: the total costs of radiology services are rising far faster than the payer's ability to meet these costs.

It was noted in the January 1996 issue of the Radiological Society of South Africa's new publication, Update, that some US radiological groups have seen their incomes drop $50 \%$ in recent years, due to an estimated $28 \%$ oversupply of board-certified radiologists, and also to the creation of large networks or radiology practices.
The net result is the consolidation of radiology practices to reduce costs, achieve economies of scale and retain income for radiologists. If a radiology practice loses a contract because it bid too high a capitation rate to payers in a large geographical area or population, that practice could go under.

One US radiology practice was approached by a large (over 70000 members) managed care organisation (MCO) to capitate diagnostic radiology and magnetic resonance imaging (MRI). It got proactive, collected data on the use rates of radiology services, designed its own utilisation management software based on Windows 3.1 (now used in over 40 US radiology practices) and negotiated five contracts in its region of California. It also subcontracted with another radiology practice, offering a discounted fee for service so it could cover a wider geographical area than was possible from its practice.

In South Africa and the United States, developments in teleradiology could accelerate the use of capitated contracts between payers and larger radiology practice networks. Faced with annual increases of over $20 \%$ in radiology costs, US employers such as the 23000 employee Harris Corporation in Florida contracted with one of the new US radiology entrepreneurs (Medical Technology Transfer Corp - MTT) which has linked to the University of California Los Angeles Medical Center. Harris has a 7-year contract with MTT based on a flat capitation fee related to 1993 prices, effectively holding Harris' radiology costs constant until 2002, saving Harris about US\$10 million over 7 years.

Four facets of this contract may be relevant to radiologists outside the USA. First, radiology costs become more predictable for the payer. Second, UCLA provides its expertise in remote areas in CT, MRI, ultrasound or X-ray via electronic links, providing an opinion immediately for critical 
cases or by express mail for less critical cases. Third, while such telemedicine is experimental in many nations, the radiologist's opinion is attracting a teleradiology reimbursement. Aetna and MetLife, two large commercial insurance companies, are also customers of MTT - and they reimburse for such a service. So does the US Medicare system, which has a 3-year project under way in four US states. Fourth, teleradiology may enable radiologists to reduce the costs of any unnecessary surgery and inappropriate hospital admissions, particularly in remote areas which may lack specialist expertise in diagnostic imaging. One region of Kaiser Permanente now sends digital CT and MRI images to the homes of the radiologists so they don't need to come to the hospital to given an expert opinion. An- other large HMO in New Mexico allows primary care practitioners to send electrocardiograms and $\mathrm{X}$-rays to specialists and then use video-conferencing for further consultations.

In nations with large, inhabited rural areas lacking health facilities (such as the United States and South Africa), developments in teleradiology will encourage the larger urban hospitals to employ fewer inhouse radiologists because a wide range of radiology sub-specialists will now be available on-line. Professional fees will decline in hospitals and the quality of and access to radiological services will increase in previously deprived areas. Hospital radiology services may again become revenue centres rather than high cost centres.

In this environment, government and large payers are likely to push for capitated contracts for radiology - and also for pathology, cardiology and other procedural specialties. In such an environment, solo practitioners will be at a significant disadvantage if they have only one or two diagnostic imaging modalities, or their equipment is outdated, or they lack access to subspecialist radiologist expertise such as neuroradiology.

At a 2-day training program on capitation contracting that our company implemented for 120 radiologists in Johannesburg from 2-3 March 1996, the message from the Radiological Society was clear: rationalise on a regional basis as managed care emerges in South Africa, as funders introduce managed fee-for-service or capitation methods of payment.

\section{COMPANY NEWS}

\section{Teleradiology system supports extensive multi-tasking}

\begin{abstract}
T ecmed Imaging (Pty) Limited recently launched their Teleradiology System incorporating TeleMAX ${ }^{\mathrm{TM}}$ software from Cemax-Icon. The Cemax-Icon ${ }^{\mathrm{TM}}$ product has been chosen by over 5000 users worldwide, as well as supporting over 1500 network users due to its superb image acquisition, transfer, display and image quality. This FDA approved and DICOM 3 Compatible TeleRadiology System supports extensive multi-tasking by allowing acquisition and display, simultaneous with image transfer between two stations. Performance is further enhanced by using state of the art Macintosh Power PC Systems, supporting hi-resolution 1200 x 1600 portrait or $1600 \times 1200$ landscape displays in a single or dual monitor con-
\end{abstract}

figuration. Higher resolutions display systems are also available.

The acquisition software supports film digitization via a high resolution $2000 \mathrm{x}$ $2500 \times 12$ bit laser scanner, for all film sizes up to $14 \times 17$ inch $(35 \times 43 \mathrm{~cm})$. The laser film digitizer may be complemented by an interface supporting up to four video modalities like CT, MRI, ultrasound, nuclear medicine as well as DSA units. Image transmission via local or wide area networks is available.

Display software functionality includes optimising brightness and contrast settings, magnifying glass, roam, paging, multiframe display, video invert, reporting and many more, giving the radiologist extensive functionality to aid the diagnosis.
The fail-safe telecommunications software package provides automatic re-dial, if line is lost, user selectable compression ratios for either Lossless or Lossy image compression, as well as a telecommunications $\log$ file used as a permanent record of all transmission activities. Send, receive and retrieve communications are available for receiving or retrieving images from other display stations. Support for standard telephone lines, ISDN or Diginet connections, allows customised Medical Network Solutions for all sites.

The system also forms the basis for an extensive departmental network, allowing connection into Computer Radiography (CR) Systems, as well as allowing upgrades for a complete PACS system. Installed base equipment can be connected to a DICOM 3 Network via customised DICOM 3 Gateways.

For further details, please contact Tecmed(Pty)Ltd on (011)315-4874. 